\title{
RASTREANDO AUSENCIAS: LA HIPÓTESIS DEL ABANDONO DEL USO DE LOS RECURSOS MARINOS EN EL MOMENTO ECUESTRE EN LA PATAGONIA CONTINENTAL
}

\author{
EDUARDO J. MORENO"Y BLANCA A. VIDELA"
}

\begin{abstract}
RESUMEN
Durante las investigaciones en la costa norte de Santa Cruz, se ha detectado una contradicción entre datos arqueológicos y etnohistóricos, especialmente para los siglos XVIII y XIX. En efecto, los datos arqueológicos indican un uso intensivo de los recursos marinos, en tanto que los etnohistóricos no hacen referencia a este fenómeno. Para resolver esta contradicción se plantea la hipótesis de que existió un abandono de la utilización de estos recursos en el periodo mencionado y se la pone a prueba en dos escalas. La primera escala es la comparación regional mediante la información radiocarbónica y faunística publicada por diversos autores para Patagonia Continental y la otra escala es local, analizando el cambio entre sitios y componentes anteriores y posteriores al contacto espacialmente próximos entre si. Para este último caso se utilizo la información de los sitios cañadón Giménez, bahía Solano 13 y bahía Solano 16. Estos primeros resultados avalan parcialmente la hipótesis planteada.
\end{abstract}

PALABRAS CLAVE: Cazadores recolectores. Pedestre-Ecuestre. Patagonia.

\section{TRACING ABSENCES: THE HYPOTHESIS OF THE MARINE RESOURCES ABANDONMENT DURING THE EQUESTRIAN PERIOD IN CONTINENTAL PATAGONIA}

\begin{abstract}
During the research on the northern coast of Santa Cruz province it has been noticed a contradiction between the archeological and ethnohistorical data, especially those referred to the 18th and 19th centuries. In fact, the archeological data suggest an intensive use of the marine resources, while the ethnohistorical ones are not related to this activity. To sort out this contradiction it was hypothetically suggested that these resources were abandoned in the above mentioned period. This hypothesis is submitted to tests at two scales: the first scale implies comparing radiocarbon and fauna information collected throughout the area and published by different authors; the second scale is local and consists of analyzing the changes between sites and components close in space to each other before and after

\footnotetext{
Centro Nacional Patagónico (CONICET), UNPSJB. Boulevard Brown 2825, Puerto Madryn (Chubut), Argentina. julianemoreno@yahoo.com

* UNPA-UACO. Ruta 3 S/N, Caleta Olivia (Santa Cruz), Argentina. blancavidela@yahoo.com
} 
the contact. For this last case information from cañadón Giménez, bahía Solano 13 and bahía Solano 16 was used. These preliminar results partially support the hypothesis proposed.

KEY WORDS: Hunter-gatherers. Equestrian-pedestrian. Patagonia.

\section{EL PROBLEMA}

Este trabajo representa la continuación del proyecto "Arqueología de la costa norte de Santa Cruz", iniciado en el año 1987. Uno de los resultados más destacado de este proyecto fue que se pudo determinar el uso intensivo de la costa y sus recursos en el Holoceno Tardío. Esto se evidencia en la alta densidad de sitios en la franja costera en contraposición al interior, y la disposición de éstos con relación a los recursos marinos (especialmente bancos de moluscos); además, estos sitios son, en su gran mayoría, concheros más o menos densos. Entre los restos óseos de alimentación allí hallados predominan los animales marinos (en especial, lobos marinos y cormoranes). Por otro lado, existen al menos dos tipos de artefactos relacionados con la captura de estos recursos: arpones de punta móvil y rompecráneos. (Moreno y Castro 1995-1996, Moreno 2003). En otros lugares de la costa continental se ha detectado la utilización de recursos marinos (ver bibliografía específica en anexo, para mas información puede verse Cruz y Caracotche 2006), si bien, quizás, con variable intensidad.

Es de notar que esta información se contradice francamente con las fuentes escritas, ya que en ellas se encuentran muy pocos registros sobre la utilización de recursos marinos. De acuerdo con el estudio de fuentes provenientes de los siglos XVI y XVII, esta utilización presenta un fuerte sesgo estacional. Las descripciones corresponden al otoño y el invierno, mientras que la mayoría de los sitios arqueológicos en los que pudo determinarse la estacionalidad, corresponden a la primavera y/o el verano, aún considerando las dificultades para la determinación invernal (Moreno 2003:86-89). En consecuencia, es lógico que los viajeros no hayan registrado la utilización de los recursos marinos (Moreno e Izeta 1999). Por otro lado, se pudo verificar que para la costa norte de Santa Cruz, la información escrita sobre los dos primeros siglos de contacto sólo se basa en los relatos de las expediciones de Magallanes, Drake y Narborought (Moreno 2003:114-128).
Con respecto a los siglos XVIII y XIX -es decir el momento en que la sociedad indígena adopta el caballo- la situación es distinta. Sobre esa época contamos con numerosos relatos de viajes realizados en todas las estaciones, con estadías prolongadas y con mejores descripciones de la vida de los grupos indígenas de la Patagonia. Con todo, sólo se registra la utilización de estos recursos en casos aislados. Por esta razón se postuló que en aquel momento los recursos marinos habrían sido abandonados (Moreno 2003:149-150). Este problema ya había sido detectado por Gómez Otero, quien señaló que su modelo de uso de la costa se restringe al momento pre-ecuestre (Gómez Otero 1995:88).

En este trabajo se pondrá a prueba la siguiente hipótesis: entre los momentos ecuestre y pre-ecuestre se produjeron cambios referidos al abandono de los recursos marinos. Sin embargo, esto no significa postular que la adopción del caballo haya sido el factor causal. Se trata de determinar si esos cambios realmente se produjeron, dejando la o las causas para trabajos futuros.

Con este fin, el primer paso consistió en elaborar una serie de expectativas arqueológicas acotadas a la zona costera, para el registro ecuestre en relación con el pre-ecuestre. Estas expectativas eran: A) una disminución de la frecuencia de sitios, B) un menor tamaño de los sitios y una menor densidad de artefactos en éstos, C) una baja frecuencia de restos (óseos o malacológicos) de animales marinos. De estas tres expectativas, la tercera es la que mide de modo más directo el fenómeno en estudio; las otras dos lo hacen indirectamente al vincular el abandono de los recursos marinos con la intensidad de ocupación del área; es decir, cuando no se utilizan los recursos del mar, la costa está menos ocupada.

Se plantearon dos líneas de trabajo: una, a escala regional, que consistió en el análisis de los sitios datados en la costa patagónica continental destinada a evaluar la expectativa A y C; y la otra, a escala local, referida al análisis de sitios -cañadón Giménez y bahía Solano 13 y 16- en los que se 
detectaron ocupaciones atribuibles a los momentos anterior al ecuestre y al ecuestre mismo (o próximos a él, ver abajo) y, por ello, aptos para verificar las expectativas B y $\mathrm{C}$. En este último caso se pudo realizar una evaluación más detallada y, además, ubicada dentro de un mismo ambiente local; es decir, donde la disponibilidad de recursos era similar.

Un problema previo a resolver fue el de la cronología de la adopción del caballo por parte de las sociedades indígenas de Patagonia. La primera mención sobre la utilización del caballo se remonta a 1621 en el norte de Patagonia (Martinic 1995:74), para la Patagonia central hay un relato sobre el hallazgo de un esqueleto de caballo en proximidades de Puerto Deseado en 1699 (Martinic y Quiroz 1989-90:30). Con respecto al sur de la región en 1704 la expedición de Carman contacta en proximidades del estrecho un grupo que aparentemente no tiene caballos. Para el mismo lugar, el primer dato irrefutable de indígenas montados se remonta a 1741 (Palermo 1988:55).

$\mathrm{Si}$ se considera que los primeros caballos fueron introducidos a Patagonia desde la llanura bonaerense, la zona de Cuyo o el actual territorio de Chile, probablemente por intercambios entre grupos (Martinic op. cit.:76), es dable esperar que su adopción ocurriera de norte a sur, o sea que puede plantarse que en 1741 todos (o casi todos) los grupos de Patagonia contaban con este animal, ya que la observación ocurre en un lugar muy próximo al límite austral de su distribución. Dado el registro de restos de caballo en 1699 en puerto Deseado, no es aventurado suponer que el establecimiento del habito ecuestre en toda la Patagonia continental se remontaría a, por lo menos, unos 10 años antes de 1741; es decir, a 1730 (220 AP), seguramente esto ocurrió antes en el norte que en el sur.

Por otro lado tiene que haber existido una transición entre el pedestre y el ecuestre, en la que seguramente coexistieron elementos de ambos, por esta razón no parece prudente extender mas atrás de 1730 el recorte temporal utilizado en este trabajo. Esto no significa que planteamos este sea el inicio de la utilización del caballo - evento situable entre el final del XVII y comienzos del XVIII (Martinic op.cit.:76) - sino que lo se intenta al tomar esta fecha es recortar un segmento temporal en el que podemos considerar que la utilización del caballo está generalizada y establecida.
De todos modos, la ampliación del límite no afectaría, en lo fundamental, las conclusiones de este trabajo.

El momento final se ubicó hacia 1880 (70 AP), fecha en que gran parte de los grupos patagónicos perdieron su autonomía como consecuencia de las campañas militares y la expansión de la ganadería, y fueron confinados en reservas o incorporados al sistema ganadero en expansión.

Con esta resolución -provisoria y aproximadadel tema cronológico, es posible pasar al problema propiamente dicho.

\section{ANÁLISIS DE ESCALA REGIONAL}

Para este análisis se consideraron 36 sitios, componentes y dataciones ${ }^{1}$ de Patagonia continental correspondiente a los últimos 2.000 años AP. Se descartaron de la muestra aquellos fechados realizados en valva, ya que aún no se cuenta con un cálculo del efecto reservorio para la totalidad de la costa, y los resultados obtenidos hasta ahora muestran una notable variabilidad (Cordero et al. 2000).

Los sitios se clasificaron en dos categorías: $\mathrm{Ch} / \mathrm{fm}$ para los concheros y/o aquellos que contenían fauna marina en cantidades apreciables; y Nch/ft, para aquellos que no tenían gran cantidad de valvas o en los cuales predominaba la fauna terrestre. En todos los casos nos basamos en la descripción dada por los autores. En algunos casos, como por ejemplo cabo Vírgenes 8, sólo se contó con una enumeración de la fauna presente debido al estadio preliminar de las excavaciones; sin embargo, como en la lista de especies predominaban las marinas (L'Heureux 2006:82), se lo incluyó en el grupo Ch/fm. El componente superior de cañadón Giménez se localiza contextualmente en el ecuestre, por lo que se lo ubicó entre 220 y 70 AP (ver abajo).

Los datos utilizados y las fuentes bibliográficas respectivas se encuentran en el anexo A.

Respecto a la primera expectativa planteada, A, se obtuvieron los datos pertinentes dividiendo las muestras de fechados en intervalos de 150 años. Cada intervalo fue numerado del 1 -el más antiguoal 14, y se contabilizaron los casos dentro de cada intervalo, según el valor central y la mayor propor-

1 En el caso de que un sitio presentara más de una datación se lo consideró como una muestra independiente. Es decir se lo contabilizo como si se tratara de otro sitio o componente. 
TABLA 1.

\begin{tabular}{|c|c|c|c|c|c|}
\hline Intervalo & Años AP & Conch/fm & no ch/ft & Total & conch/fm \% \\
\hline 1 & $2.021-2.170$ & 1 & & 1 & 100 \\
\hline 2 & $1.871-2.020$ & 1 & 1 & 2 & 50 \\
\hline 3 & $1.721-1.870$ & 0 & 0 & 0 & 0 \\
\hline 4 & $1.571-1.720$ & 4 & & 4 & 100 \\
\hline 5 & $1.421-1.570$ & 4 & 1 & 5 & 80 \\
\hline 6 & $1.271-1.420$ & 4 & & 4 & 100 \\
\hline 7 & $1.121-1.270$ & 4 & & 4 & 100 \\
\hline 8 & 971-1.120 & 2 & & 2 & 100 \\
\hline 9 & $821-970$ & 2 & & 2 & 100 \\
\hline 10 & $671-820$ & 3 & & 3 & 100 \\
\hline 11 & $521-670$ & 2 & & 2 & 100 \\
\hline 12 & $371-520$ & 2 & & 2 & 100 \\
\hline 13 & $221-370$ & 1 & 1 & 2 & 50 \\
\hline 14 & $71-220$ & 2 & 1 & 3 & 66.7 \\
\hline
\end{tabular}

ción del desvío del fechado. Se tomaron periodos de 150 años porque esa es la duración estimada del momento ecuestre (Tabla 1).

Al presentar estos datos, en la Fig. 1 se observa que no se verifica una disminución en el número de sitios, sino una fluctuación no direccional.

En cuanto a la distribución espacial de sitios tipo $\mathrm{ch} / \mathrm{fm}$ y los nch/ft, los datos se observan en la Fig. 2. Allí, el primer intervalo comienza en el 70 AP y termina en el $220 \mathrm{AP}$; es decir que corresponde al momento ecuestre. Los sitios de tipo $\mathrm{Ch} / \mathrm{fm}$ se representan con su valor central en negro; y los del tipo $\mathrm{Nch} / \mathrm{ft}$, con un recuadro vacío. Respecto al análisis de la expectativa C, la Fig. 2 muestra que la mayor cantidad de sitios no concheros o con fauna terrestre se agrupan en el periodo ecuestre.

Para poner a prueba esta relación, los datos fueron tratados como discontinuos y asignados al

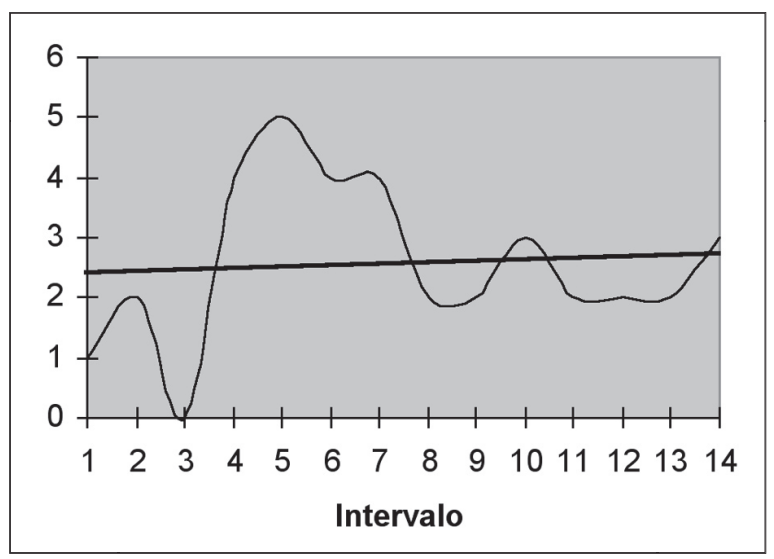

Fig. 1.

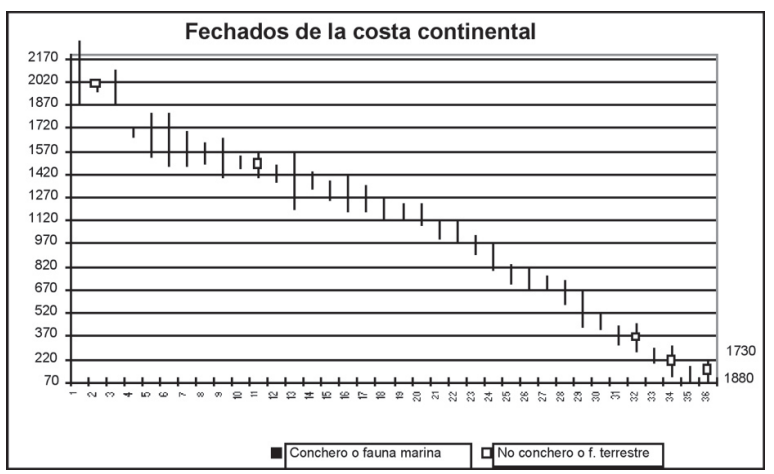

Fig. 2.

momento ecuestre o pre-ecuestre, utilizando como criterio el valor central y la mayor porción del desvío para esta asignación ${ }^{2}$. (Tabla 2).

Esto permitió utilizar la prueba de Fisher exacta, la cual arrojó una probabilidad de 0,043 (por lo tanto, menor al nivel de significación de $0,05)$. Con estos resultados es posible descartar la hipótesis nula que propone que no hay diferencias entre el ecuestre y el preecuestre. Es decir, es sig-

TABLA 2.

\begin{tabular}{|c|c|c|c|}
\hline & Nch/Ft & Ch/Fm & \\
\hline Ecuestre & 2 & 1 & 3 \\
\hline Pre-ecuestre & 3 & 30 & 33 \\
\hline & 5 & 31 & 36 \\
\hline
\end{tabular}

2 Los fechados presentados no fueron calibrados; sin embargo, se han calibrado los cinco fechados más cercanos al momento ecuestre utilizando el programa Calib 5.0.2. Esta calibración no arrojó diferencias que hicieran cambiar la asignación de ningún sitio del ecuestre al momento anterior o viceversa. 
TABLA 3.

\begin{tabular}{|c|c|c|c|}
\hline & Nch/Ft & Ch/Fm & \\
\hline $\begin{array}{c}\text { Contacto inicial } \\
(221-370)\end{array}$ & 1 & 1 & 2 \\
\hline Pre-contacto & 2 & 29 & 31 \\
\hline & 3 & 30 & 33 \\
\hline
\end{tabular}

nificativa la diferencia entre la cantidad de sitios de un tipo y otro. Para verificar si el cambio se vincula al momento ecuestre o si se trata de una tendencia más general, se realizó este mismo test comparando el período inmediatamente anterior -el cual representa el contacto inicial (221-370 AP)- con el resto (Tabla 3).

Los resultados obtenidos indicaron una probabilidad de 0,17, lo cual no permite rechazar la hipótesis nula. Es decir, no hay diferencias significativas en la cantidad de sitios de uno y otro tipo entre el contacto inicial y el pre-contacto.

\section{ANÁLISIS DE ESCALA LOCAL}

\section{a) El sitio cañadón Giménez}

En relación con las expectativas planteadas se realizó una prospección en las proximidades de la ciudad de puerto Deseado, debido al atractivo que pudo haber tenido la compañía pesquera establecida allí, a finales del siglo XVIII. Gracias a información recopilada entre la población actual, se descubrió que en la zona de cañadón Giménez, aficionados locales habían detectado un sitio que contenía materiales eurocriollos e indígenas. Este material se encuentra depositado en el museo del colegio San José, de esa localidad. Entre los elementos se encuentran cuentas de vidrio y metales, restos óseos de pinnípedos, puntas de proyectil, fragmentos de cerámica e, incluso, arpones de hueso.

La prospección permitió detectar un sitio

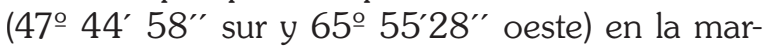
gen este del cañadón, a unos 50 metros del mar y sobre un antiguo cordón litoral. El sitio reveló una intensa alteración debido a que fue destruido en su mayor parte por maquinas viales (Fig. 3). El trabajo inicial consistió en recolecciones superficiales y refilamiento de perfiles a fin de detectar áreas aún conservadas. Las recolecciones permitieron hallar algunos restos óseos de caballo europeo mezclado

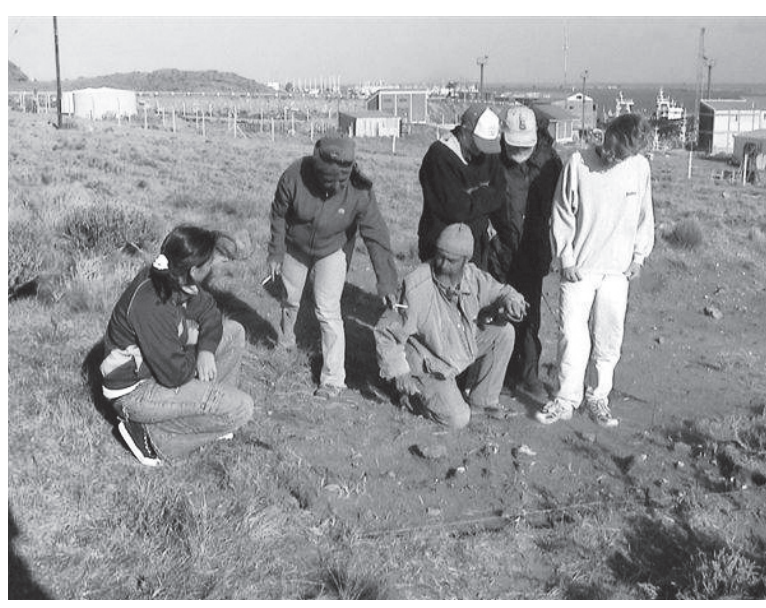

Fig. 3. Sitio Cañadón Giménez. Al fondo se puede ver la ciudad de Puerto Deseado. El grupo de personas esta ubicadas sobre el sector alterado del sitio.

con otros materiales. El refilamiento de los perfiles indicó que quedaban algunos reducidos sectores sin alterar.

Inicialmente se realizó un sondeo (sondeo 1) de un metro de lado a fin de verificar la estratigrafía. Se halló una capa superior limo-arenosa poco consolidada de $9 \mathrm{~cm}$. de espesor, por debajo de la cual había una capa de limo compactado que llegaba hasta los $27 \mathrm{~cm}$. Por debajo se halló una capa arcillosa en la que no se profundizó. En la capa superior se hallaron pocos materiales arqueológicos, pero en la capa consolidada y, a partir de los 15 $\mathrm{cm}$., apareció un claro nivel de conchero asociado a materiales líticos, cerámicos y óseos. En ninguna de las dos capas de esta cuadricula se halló material de origen europeo. Esto, sumado a la composición faunística y a la presencia de cerámica, sugirió que la capa consolidada pertenecía al momento anterior al contacto. Por lo tanto, se realizaron una serie de sondeos a fin de verificar la existencia de materiales en la capa superior en otros lugares del sitio. En todos los casos se excavó sólo hasta llegar al sedimento consolidado. Se realizaron un total de 11 sondeos de este tipo (Fig. 4).

En el sector noreste del sitio, se verificó un mayor desarrollo del sedimento limo-arenoso sin consolidar, intercalado con una capa más o menos continua de ceniza volcánica. Por esta razón, se amplio la excavación a dos cuadriculas de 1 x $1 \mathrm{~m}$., con tres anexos de 0,5 x 0,5 m., teniendo en cuenta la posibilidad de ampliar la excavación evitando 


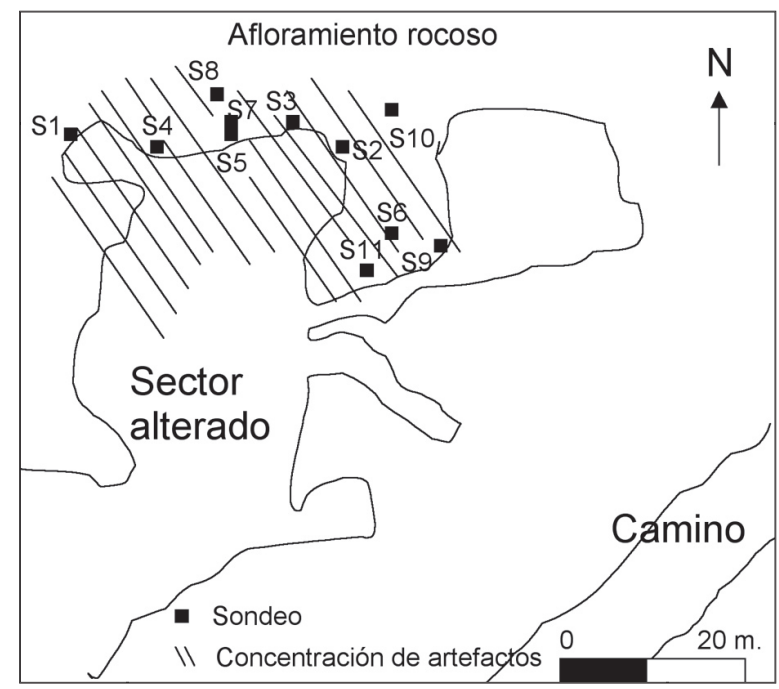

Fig. 4. Sitio Cañadón Giménez. Planta del sitio y sondeos.

los sectores alterados. Los resultados permitieron recuperar una muestra de materiales consistentes en restos óseos, artefactos líticos, escasas valvas y algunos pequeños fragmentos de metal además de fragmentos de cerámica no indígena. También se hallaron improntas de cuevas de roedores, tanto en el sedimento blando como en el techo del sedimento más consolidado.

A 1,5 metros al norte de estas cuadrículas, se excavó otra de un metro por un metro. Allí, dentro de la capa de ceniza, se hallaron vidrios rotos in situ y escasos materiales de otro tipo. En este caso, también se detectaron improntas de cuevas que contenían fragmentos de plástico, lo cual revela que el sitio fue alterado. En la mayoría de los casos se intentó diferenciar al máximo esta capa superior tratando de separar los materiales según provinieran de la parte inferior o superior de la capa de cenizas; sin embargo, debido a lo escaso de la muestra, fue analizado sin diferenciarlo. Estos datos se encuentran resumidos en el Anexo B.

Discusión e interpretación del sitio:

En el sitio pudieron separarse dos componentes estratigráficamente diferenciados: el superior en una matriz limo-arenosa poco compactada (capa 1); y el componente inferior en matriz limosa compacta (capa 2). Entre el final de la capa 1 y el inicio de la aparición de materiales en la capa 2 existe un hiato de unos 6 centímetros.
En el resto de los sondeos sólo se excavó la capa 1. Esto permite afirmar que todos los materiales de los sondeos 2 al 11 corresponden al componente superior, salvo intrusiones debidas a la existencia de numerosas cuevas de roedores en el sitio.

Respecto de la cronología de los componentes, el componente superior presenta materiales de procedencia europea -cerámica vidriada, vidrios, metales, carbón de piedra e, incluso, plásticos. En general, estos últimos fueron hallados con vinculación a cuevas y, en un único caso, muy cerca de la superficie. Entre los artefactos metálicos se incluye un proyectil identificado como .45 ACP. El hecho de haber sido disparado hace suponer que su presencia en capa puede deberse a que impactó en el lugar. Entre los vidrios se cuenta un ejemplar de vidrio plano y otros ocho fragmentos curvos que fueron hallados fracturados in situ, seis de estos fragmentos pudrieron ser remontados.

En la cuadricula 6 se halló, además, un fragmento de metapodio perteneciente a un mamífero terrestre grande. Sin embargo, puede descartarse que corresponda a un guanaco. Por su tamaño solo podría pertenecer a un caballo o a un vacuno. Esto permite suponer que este componente pertenece al momento ecuestre o es posterior a él.

Un rasgo notable de esta capa es que no se hallaron restos óseos de liebre europea ni de oveja. Ambos animales fueron introducidos con posterioridad a 1880 y desde entonces, han sido muy abundantes. Sólo se hallaron excrementos de oveja en la superficie y en los primeros centímetros de excavación. Todo esto indica que el depósito se formó entre el período inicial del momento ecuestre y con anterioridad a la fundación de Puerto Deseado (1880); aunque, posteriormente, sufrió alteración como consecuencia de la acción de roedores. Éstos mezclaron fragmentos de plástico y, quizá, los otros elementos; es decir, los fragmento de carbón de piedra y el vidrio plano.

En cuanto al segundo componente, se dató una muestra de valva en $1.470 \pm 70$ años C-14 A.P. (LP 1800). Si se considera el efecto reservorio como de 400 años, se llegaría a unos $1.070 \pm 70$ años. El rango de la edad calibrada con una probabilidad del 95\% ( \pm 2 sigmas) sería de entre 779 - 1.056 años cal AD. Respecto al problema en discusión, lo relevante en este caso es que esta ocupación no corresponde al momento ecuestre (Fig. 5). 


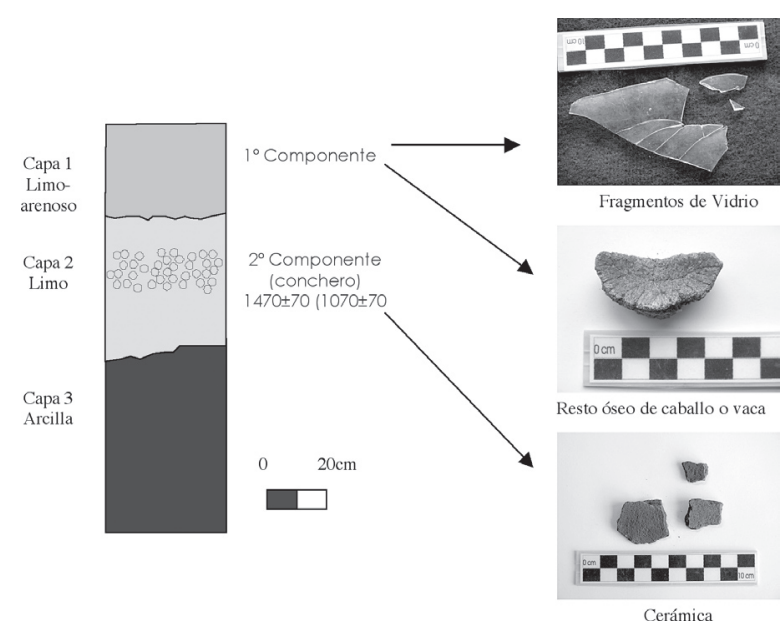

Fig. 5. Perfil del Sitio Cañadón Giménez.

b) Los sitios de bahía Solano

Se trata de una localidad ubicada a pocos kilómetros al norte de la ciudad de Comodoro Rivadavia, en la que se han detectado gran cantidad de sitios (para una descripción más completa ver Borrero y Caviglia 1978; Caviglia y Borrero 1978 y Caviglia et al. 1982). De estos, se han fechado los sitios BS 13 y BS 16, los cuales han sido elegidos para este trabajo. El primero de ellos es un sitio datado en $205 \pm 95$; y el segundo, en $2.954 \pm 195$ (capa III). Al igual que los componentes de cañadón Giménez, estos sitios presentan la ventaja de encontrase relativamente próximos y, además, se cuenta con una descripción detallada de su estratigrafía y su composición faunística y artefactual.

El sitio BS 13 está parcialmente comprendido dentro del ecuestre, aunque una parte considerable del desvío cae fuera de ese período. Por otro lado, no se hallaron en él elementos europeos que permitieran ajustar su cronología, si bien se encontraron fragmentos de cerámica y una punta lítica de proyectil. El primero de estos artefactos no aparece mencionado en las crónicas del momento ecuestre, en tanto que el segundo lo es con poca frecuencia. Según los datos recopilados por Embon (1950:213), las puntas líticas son mencionadas hasta 1833. Pese a esta duda, como ya se señaló, se considera que este sitio corresponde al momento ecuestre, ya que su valor central -y la mayor parte del desvió (1 sigma)- se encuentra dentro de este período. En todo caso, representaría un período ecuestre inicial, mas tomando en consideración que el sitio se encuentra al norte de Puerto Deseado en donde el registro de caballos se remonta a 1699 (Martinic y Quiroz 1989.90).

Los datos aquí presentados provienen de la publicación de Caviglia et al. (1982), salvo los referidos al NISP de ambos sitios (datos que fueron brindados gentilmente por el Licenciado Sergio Caviglia).

c) Resultados del análisis a escala local

Los resultados de ambos sitios se encuentran resumidos en el Tabla 4.

Como se puede ver en las Fig. 6 y 7, la cantidad de materiales por metro cuadrado disminuye entre el componente anterior al contacto (sea el componente inferior de cañadón Giménez y bahía Solano 13) y el componente más reciente. Esta disminución es mucho más notable en la relación valvas/superficie que en las demás. En otras palabras, si tomamos la cantidad de artefactos, valvas y restos óseos del momento anterior al contacto como equivalentes al 100\%, la reducción en el componente histórico de cañadón Giménez es del 76,1\% en el caso de los artefactos; del 74,7 \% para los restos óseos, y del 88,6 \% para las valvas. En bahía Solano la reducción es del 58,8 \% para los artefactos, del 63,6 \% para el óseo y de 77,4\% para las valvas. Como se ve en los dos casos, la mayor reducción se produce con respecto a las valvas; o sea, es un indicador directo de la utilización de los recursos marinos.

TABLA 4

\begin{tabular}{|c|c|c|c|c|c|c|c|c|c|c|c|}
\hline Cañadón Giménez & Valvas & artefactos $^{*}$ & val/art & óseo & sup. $\left(\mathrm{m}^{2}\right)^{* * *}$ & Art $/\left(\mathrm{m}^{2}\right)$ & $\mathrm{val} /\left(\mathrm{m}^{2}\right)$ & óseo $/\left(\mathrm{m}^{2}\right)$ & lama & $\mathrm{pinn}$. & $\mathrm{pinn} \%$ \\
\hline Primer Comp.(hist.) & 395 & 610 & 0,6 & 23 & 7 & 87.1 & 56.4 & 3.3 & 9 & 4 & 30.8 \\
\hline Segundo comp & 494 & 366 & 1,3 & 13 & 1 & 366.0 & 494.0 & 13.0 & 3 & 10 & 76.9 \\
\hline Bahía Solano & Valvas & artefactos & art/val & óseo & sup. $\left(\mathrm{m}^{2}\right)^{* *}$ & $\mathrm{art} /\left(\mathrm{m}^{2}\right)$ & $\mathrm{val} /\left(\mathrm{m}^{2}\right)$ & óseo $/\left(\mathrm{m}^{2}\right)$ & lama & $\mathrm{pinn}$. & $\mathrm{pinn} \%$ \\
\hline BS 13 (hist.) & 72 & 217 & 0,3 & 12 & 5.5 & 39.5 & 13.1 & 2.2 & 12 & 0 & 0.0 \\
\hline BS 16 (C III) & 232 & 381 & 0,6 & 24 & 4.0 & 95.3 & 58.0 & 6.0 & 16 & 47 & 74.6 \\
\hline
\end{tabular}

"líticos, vidrio, metal y cerámica; "determinables anatómicamente; " superficie excavada. 


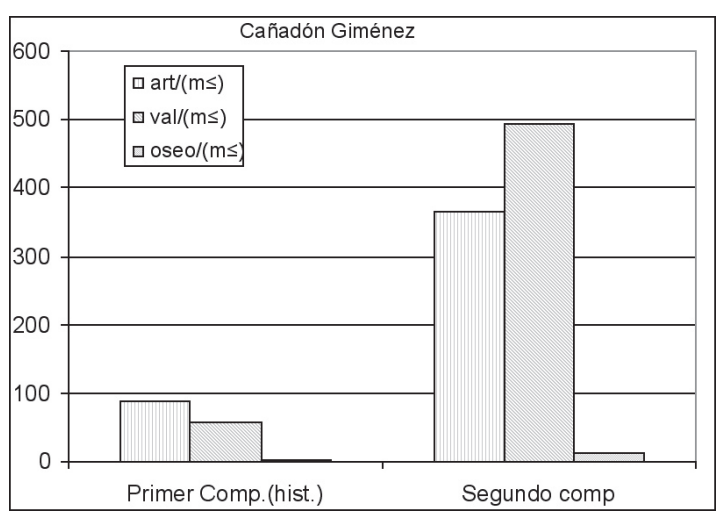

Fig. 6.

Esta interpretación conlleva el problema de que podemos estar comparando lapsos temporales de distinta duración. Es decir, estaríamos comparando los 150 años de duración del momento ecuestre con los potenciales miles de años de duración de los componentes anteriores (ya que no disponemos de fechados de techo y base). Esta posibilidad de comparación puede argumentarse de dos maneras: por un lado, puede señalarse que el hecho de tratarse de lentes de concheros de unos 10 a 20 $\mathrm{cm}$. de espesor sugiere una formación rápida de los componentes pre-cuestres. En el caso de bahía Solano los sitios (en términos generales, no solo los aquí tratados) fueron interpretados por los autores como "rápidos pasajes" (Borrero y Caviglia 1978:9). Por otro lado, si la diferencia fuera sólo temporal, todos los valores (artefactos, valvas restos óseos) deberían descender en proporción similar. Esta condición no se cumple ya que, como se señaló más arriba, las valvas se reducen en mayor proporción que los demás elementos. Esta conclusión

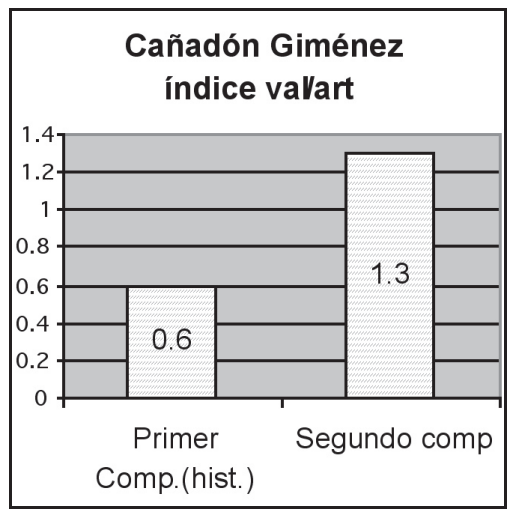

Fig. 8.

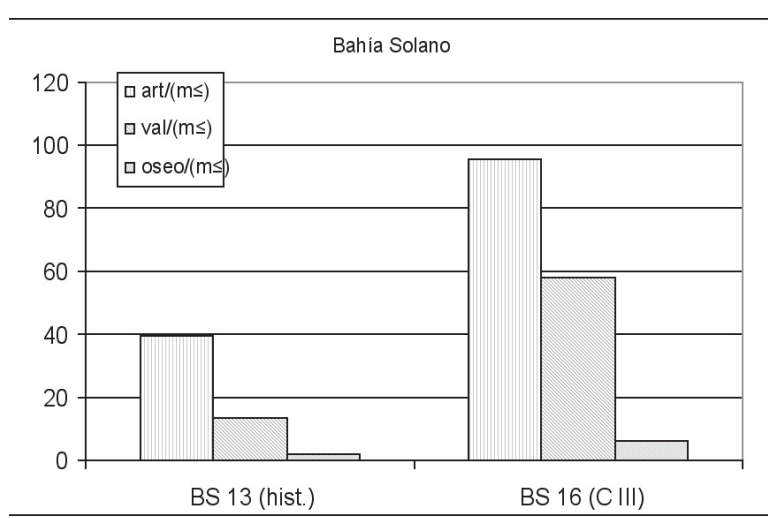

Fig. 7.

se vuelve más patente cuando se elabora un índice de valvas/artefactos. La ventaja de este índice es que no depende del tiempo de formación del depósito. En las Fig. 8 y 9 se puede verificar que en los componentes históricos, la cantidad de valvas disminuye prácticamente a la mitad en relación a los artefactos. Esto indica que en las diferencias hay un factor distinto del tiempo implicado.

Con respecto a la fauna de vertebrados presentes, en el caso del sitio cañadón Giménez y considerando sólo los restos de guanacos y pinnípedos (estos animales fueron seleccionados por ser por mucho los más abundantes en el sitio y representativos de los ambientes terrestres y marinos respectivamente), en el componente superior el NISP de los primeros, representa el $70 \%$ de la muestra; mientras que, en el caso del componente inferior, representa sólo el 23 \% (Tabla 5). Aplicando el test de Fisher exacto el nivel de significación es de 0,021; o sea, menor a 0.05 , lo cual permite descartar la hipótesis nula de ausencia de cambios.

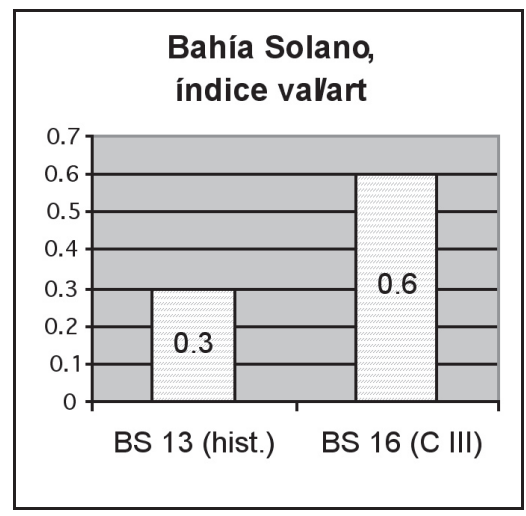

Fig. 9. 
TABLA 5. Cañadón Solano.

\begin{tabular}{|c|c|c|c|}
\hline & Lama & Pinnípedos. & Total \\
\hline Primer Comp.(hist.) & 9 & 4 & 13 \\
\hline Segundo Comp. & 3 & 10 & 13 \\
\hline & 12 & 14 & 26 \\
\hline
\end{tabular}

TABLA 6. Bahía Solano.

\begin{tabular}{|c|c|c|c|}
\hline & Lama & Pinnípedos & Total \\
\hline BS 13 (hist.) & 12 & 0 & 12 \\
\hline BS 16 (C III) & 16 & 47 & 63 \\
\hline & 28 & 47 & 75 \\
\hline
\end{tabular}

Esta misma tendencia -si bien más marcadase manifiesta en los sitios de la localidad de Bahía Solano. En este caso, al aplicar el mismo test (Tabla 6) el nivel de significación es de 0,000001.

En la Fig. 10 y 11 se puede observar este resultado expresado en valores absolutos.

Se destaca que en todos los sitios pre-ecuestres de la costa norte de Santa Cruz y la costa sur del Chubut, es normal registrar una proporción de pinnípedos superior al 70 \% con relación a los guanacos (Moreno 2003:85).

\section{CONCLUSIÓN}

En el trabajo planteamos la siguiente hipótesis: entre los momentos ecuestre y pre-ecuestre se dejaron de utilizar los recursos marinos en la Patagonia continental. Para poner a prueba esta hipótesis se generaron tres expectativas para el registro ecuestre en relación con el pre-ecuestre. Es decir que si ocurrió el abandono postulado seria esperable: A) una disminución de la frecuencia de sitios, B) un menor tamaño de los sitios y una menor densidad de artefactos en éstos y C) una baja frecuencia de restos (óseos o malacológicos) de animales marinos.

El análisis se realizó a dos escalas: regional y local.

Los resultados obtenidos en el caso de la expectativa A, analizada a nivel regional, no se cumple ya que la cantidad de sitios no disminuye en el momento ecuestre.

La expectativa B se pudo verificar parcialmente. Ya que no se disponía de datos sobre el tamaño de los dos sitios atribuibles al ecuestre, pues ambos casos (cañadón Giménez y Bahía Solano) estaban muy alterados. Sí se cumple claramente lo referido a la reducción en la densidad de artefactos en las ocupaciones posteriores al contacto.

La expectativa $\mathrm{C}$ se cumple claramente tanto en la escala regional como en la local. En el primer caso se verifica una reducción estadísticamente significativa en el número de sitios concheros o con fauna marina en el momento más tardío. En la escala local se observa una marcada reducción de la cantidad de moluscos por $\mathrm{m}^{2}$ y una acentuada disminución en la cantidad de pinnípedos en relación a los guanacos, en las ocupaciones posteriores al contacto. Como se planteo en la introducción esta expectativa es la que se vincula en forma más directa con la hipótesis.

Estos resultados permiten mantener la hipótesis que plantea una tendencia hacia el abandono del consumo de recursos marinos por parte de las poblaciones de cazadores recolectores de la Patagonia, en el momento del contacto. Pese a la escasez de sitios y a las dificultades de definición cronológica (especialmente en el caso de bahía Solano 13

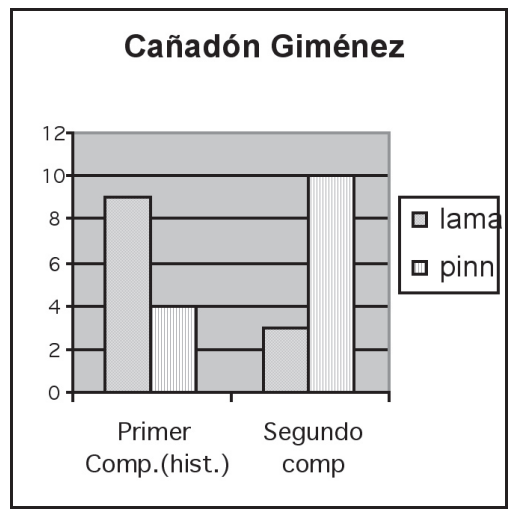

Fig. 10.

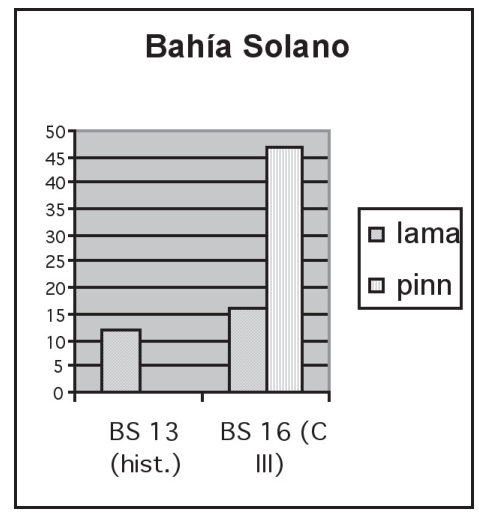

Fig. 11. 
y con respecto al inicio del momento ecuestre) y los problemas post depositacionales de cañadón Giménez, parece probable que, de acuerdo con los resultados obtenidos a escala regional, este cambio se vincule con el momento claramente ecuestre. Esto se infiere de que al comparar la frecuencia de concheros y no concheros en el contacto inicial no dio diferencias significativas, y por el contrario si fueron significativas al hacerlo con el ecuestre.

Esto coincide con los resultados obtenidos en el norte de Chubut, donde en los restos óseos humanos correspondientes al momento del contacto, se verifica una tendencia hacia las dietas terrestres (Gómez Otero 2007:305). Este fenómeno se ha vinculado explícitamente con la adopción del caballo (Gómez Otero op cit.: 420).

Esta hipótesis permite resolver la aparente contradicción entre la información arqueológica y las fuentes escritas de los siglos XVIII y XIX, ya que cada una de ellas hace referencia a momentos distintos. En ese sentido la información arqueológica sobre el momento ecuestre que aquí se presenta diluye esta contradicción.
Por supuesto, es preciso seguir sometiendo a prueba esta hipótesis, aumentando la muestra de sitios y tratando de solucionar los problemas cronológicos, tanto de los sitios arqueológicos como del paso del pedestre al ecuestre.

\section{AGRADECIMIENTOS}

La realización de este trabajo fue posible gracias al subsidio otorgado por el CONICET y la UNPA. Agradecemos a Luís Borrero, Nora Franco, Isabel Cruz y Lorena L'Heureux que nos facilitaron datos en ese momento inéditos del sitio cabo Vírgenes 8, así como a Sergio Caviglia por los datos de Bahía Solano. A Rolando Gonzáles por su asesoramiento estadístico. A Mateo Martinic, Alicia Castro, Julieta Gómez Otero y a los evaluadores por las oportunas críticas a este trabajo, a Osvaldo Tola y Marcos Oliva Day por su colaboración en los trabajos de campo. Finalmente queremos agradecer a la comunidad de Puerto Deseado por la hospitalidad brindada.

ANEXO A: Sitios de la Costa Continental Patagónica, considerados en el trabajo.

\begin{tabular}{|c|c|c|c|c|c|c|}
\hline Procedencia & Localización & Laboratorio & Edad ${ }^{14} \mathrm{C}$ & $\begin{array}{l}\text { Material } \\
\text { datado }\end{array}$ & Referencias & Tipo \\
\hline Bahía Posesión 3 & $\begin{array}{l}\text { Estrecho de } \\
\text { Magallanes }\end{array}$ & Gak-8290 & $2.080 \pm 200$ & Carbón & Massone 1979:93 & $\mathrm{Ch}$ \\
\hline Cabo Vírgenes 4 & $\begin{array}{l}\text { Boca del estrecho } \\
\text { de Magallanes }\end{array}$ & GX-27864-AMS & $2.000 \pm 40$ & Hueso lama & Borrero et al. 2006 & $\begin{array}{c}\mathrm{Ft} \\
\mathrm{nch}\end{array}$ \\
\hline $\begin{array}{l}\text { Bahía Munición } \\
2 \text {, nivel } 9\end{array}$ & $\begin{array}{l}\text { Costa noroeste } \\
\text { del Estrecho }\end{array}$ & Gif-1042 & $1.990 \pm 110$ & carbón & Orquera 1986 & $\mathrm{Ch}$ \\
\hline Cabo Blanco, $60-70 \mathrm{~cm}$ & Cabo Blanco & Beta 134598 & $1.700 \pm 30$ & hueso & Moreno 2003 & $\mathrm{Ch}$ \\
\hline Bahía Munición 3 & $\begin{array}{l}\text { Costa noroeste } \\
\text { del Estrecho }\end{array}$ & Gif-1039 & $1.680 \pm 140$ & carbón & Orquera 1986:35 & $\mathrm{Ch}$ \\
\hline Bahía Posesión 3 & $\begin{array}{l}\text { Estrecho de } \\
\text { Magallanes } \\
\end{array}$ & Gak-8289 & $1.640 \pm 170$ & Carbón & Massone 1979:93 & $\mathrm{Ch}$ \\
\hline Punta Dungeness 2 & $\begin{array}{l}\text { Estrecho de } \\
\text { Magallanes } \\
\end{array}$ & Gak-8285 & $1.590 \pm 110$ & Carbón & Massone 1979:99 & $\mathrm{N}$ \\
\hline San Gregorio 5 & $\begin{array}{l}\text { Estrecho de } \\
\text { Magallanes }\end{array}$ & Gak-8292 & $1.530 \pm 120$ & carbón & Massone 1979:89 & $\mathrm{Ch}$ \\
\hline El Piche 1 & $\begin{array}{l}\text { Bahía San Blas, } \\
\text { Buenos Aires }\end{array}$ & LP-1084 & $1.500 \pm 40$ & $\begin{array}{l}\begin{array}{l}\text { Huesos de } \\
\text { pinnípedos }\end{array} \\
\end{array}$ & $\begin{array}{l}\text { Eugenio y Aldazabal } \\
2004: 692\end{array}$ & $\mathrm{Ch}$ \\
\hline Punta Guanaco 97 & $\begin{array}{l}\text { Punta Guanaco, } \\
\text { ría Deseado }\end{array}$ & LP-1648 & $1.480 \pm 70$ & $\begin{array}{l}\text { carbón } \\
\text { vegetal }\end{array}$ & Castro, et al. 2007 & $\mathrm{Ch}$ \\
\hline $\begin{array}{l}\text { Cabo Blanco 1, } \\
10-20 \mathrm{~cm}\end{array}$ & Cabo Blanco & Beta 134597 & $1.420 \pm 50$ & hueso & Moreno 2003 & $\mathrm{Ch}$ \\
\hline
\end{tabular}




\begin{tabular}{|c|c|c|c|c|c|c|}
\hline Bahía Posesión 3 & $\begin{array}{l}\text { Estrecho de } \\
\text { Magallanes }\end{array}$ & Gak-8288 & $1.380 \pm 180$ & Carbón & Massone 1979:93 & $\mathrm{Ch}$ \\
\hline $\mathrm{CCH} \mathrm{4,} \mathrm{Nivel} 65 \mathrm{~cm}$ & P. N. Monte León & LP 1583 & $1.380 \pm 50$ & carbón & $\begin{array}{l}\text { Caracotche et } \\
\text { al. } 2006\end{array}$ & $\mathrm{Ch}$ \\
\hline $\mathrm{CCH} 4$, Nivel 85-93 cm & P. N. Monte León & LP 1609 & $1.320 \pm 60$ & carbón & $\begin{array}{l}\text { Caracotche et } \\
\text { al. } 2006\end{array}$ & $\mathrm{Ch}$ \\
\hline Bahía Posesión 3 & $\begin{array}{l}\text { Estrecho de } \\
\text { Magallanes }\end{array}$ & Gak-8287 & $1.290 \pm 110$ & Carbón & Massone 1979:93 & $\mathrm{Ch}$ \\
\hline San Gregorio 5 & $\begin{array}{l}\text { Estrecho de } \\
\text { Magallanes }\end{array}$ & Gak-8291 & $1.260 \pm 80$ & carbón & Massone 1979:89 & $\mathrm{Ch}$ \\
\hline Cabo Vírgenes 6 & $\begin{array}{l}\text { Boca del estrecho } \\
\text { de Magallanes }\end{array}$ & GX-25772 & $1.190 \pm 60$ & carbón & $\begin{array}{l}\text { Barberena et } \\
\text { al. 2004:424 }\end{array}$ & Fm \\
\hline Cabo Vírgenes 6 & $\begin{array}{l}\text { Boca del estrecho } \\
\text { de Magallanes }\end{array}$ & Beta-144998 & $1.170 \pm 50$ & carbón & $\begin{array}{l}\text { Barberena et } \\
\text { al. 2004:424 }\end{array}$ & $\mathrm{Fm}$ \\
\hline Cabo Vírgenes 6 S3 & $\begin{array}{l}\text { Boca del estrecho } \\
\text { de Magallanes }\end{array}$ & Beta-144999 & $1.160 \pm 70$ & $\begin{array}{l}\text { Hueso } \\
\text { pinnípedo }\end{array}$ & $\begin{array}{l}\text { Barberena et } \\
\text { al. 2004:424 }\end{array}$ & $\mathrm{Fm}$ \\
\hline CEM02LPM & Punta Bustamante & LP 187 & $1.060 \pm 50$ & hueso & Mansur 2007 & $\mathrm{Fm}$ \\
\hline Cabo Vírgenes 2 & $\begin{array}{l}\text { Boca del estrecho } \\
\text { de Magallanes }\end{array}$ & GX-25276-G & $1.050 \pm 70$ & \begin{tabular}{|l} 
Hueso \\
pinnípedo
\end{tabular} & $\begin{array}{l}\text { Barberena et } \\
\text { al. 2004:424 }\end{array}$ & $\mathrm{Fm}$ \\
\hline Cabo Blanco 2, CS & Cabo Blanco & Beta 134599 & $960 \pm 60$ & hueso & Moreno 2003 & $\mathrm{Ch}$ \\
\hline HST01AM & Punta Bustamante & LP 454 & $890 \pm 90$ & Carbón & Mansur 2007 & $\mathrm{Fm}$ \\
\hline Restinga Ali & $\begin{array}{l}\text { Centro de golfo } \\
\text { San Jorge }\end{array}$ & LP-511 & $770 \pm 60$ & Carbón & $\begin{array}{l}\text { Arrigoni y } \\
\text { Paleo } 1991\end{array}$ & $\mathrm{Ch}$ \\
\hline Punta Bustamante & Punta Bustamante & LP 479 & $750 \pm 70$ & Carbón & Mansur 2007 & $\mathrm{Fm}$ \\
\hline CEM04CAN & Punta Bustamante & LP 201 & $710 \pm 40$ & Carbón & Mansur 2007 & $\mathrm{Fm}$ \\
\hline Restinga Norte (SIT 6) & P. N. monte León & LP 1526 & $650 \pm 75$ & carbón & $\begin{array}{l}\text { Caracotche et } \\
\text { al. } 2006\end{array}$ & $\mathrm{~N}$ \\
\hline Bahía Posesión 3 & $\begin{array}{l}\text { Estrecho de } \\
\text { Magallanes }\end{array}$ & Gak.8286 & $550 \pm 110$ & Carbón & Massone 1979:93 & $\mathrm{Ch}$ \\
\hline La Armonía Casco & Península Valdés & LP-1001 & $460 \pm 40$ & $\begin{array}{l}\text { Huesos de } \\
\text { guanaco }\end{array}$ & $\begin{array}{l}\text { Gómez Otero et } \\
\text { al. 2002:19 }\end{array}$ & $\mathrm{Ch}$ \\
\hline $\begin{array}{l}\text { Los Abanicos } \\
1, \text { fogón } 1\end{array}$ & Península Valdés & LP-889 & $380 \pm 60$ & carbón & $\begin{array}{l}\text { Gómez Otero y } \\
\text { Suarez, 1999:396 }\end{array}$ & $\mathrm{Ch}$ \\
\hline Punta Dungeness 2 & $\begin{array}{l}\text { Estrecho de } \\
\text { Magallanes }\end{array}$ & Gak-8284 & $360 \pm 90$ & Carbón & Massone 1979:99 & $\mathrm{N}$ \\
\hline Cabo Vírgenes 8 & $\begin{array}{l}\text { Boca del estrecho } \\
\text { de Magallanes }\end{array}$ & GX-27868-AMS & $240 \pm 40$ & \begin{tabular}{|l}
$\begin{array}{l}\text { Hueso } \\
\text { guanaco }\end{array}$ \\
\end{tabular} & Borrero et al. 2006 & $\mathrm{Fm}$ \\
\hline Bahía Solano 13 & Golfo San Jorge & $\mathrm{I}-11795$ & $205 \pm 95$ & carbón & Caviglia et al. 1982 & Nch \\
\hline Cabo Vírgenes 8 & $\begin{array}{l}\text { Boca del estrecho } \\
\text { de Magallanes }\end{array}$ & GX-25774 & $120 \pm 55$ & Carbón & Borrero et al. 2006, & $\mathrm{Fm}$ \\
\hline Cañadón Giménez CS & $\begin{array}{l}\text { Costa norte de } \\
\text { Santa Cruz }\end{array}$ & $* * * * * * * *$ & ecuestre & contextual & En este artículo & Nch \\
\hline
\end{tabular}


ANEXO B: Materiales del Sitio cañadón Giménez.

\begin{tabular}{|c|c|c|c|c|c|c|c|c|c|c|c|c|c|c|}
\hline Sondeo & Capa & Lapas & Mitilidos & Otros & Total & $\begin{array}{l}\text { D. de } \\
\text { talla }\end{array}$ & $\begin{array}{c}\text { A. } \\
\text { líticos }\end{array}$ & Óseos & $\begin{array}{l}\text { C. } \\
\text { ind.* }\end{array}$ & $\begin{array}{l}\text { C. } \\
\text { eu. }\end{array}$ & Metal & Vidrio & Otros & Total \\
\hline Sondeo 1 & $\begin{array}{l}\text { Capa } 2 \\
\text { conchero }\end{array}$ & 323 & 170 & 1 & 494 & 356 & 5 & 59 & 5 & & & & 1 & 434 \\
\hline Sondeo 1 & Capa 1 & 3 & & & 3 & 28 & & & & & & & & 28 \\
\hline Sondeo 2 & Capa 1 & 4 & 2 & & 6 & 29 & 5 & 31 & & & & & 1 & 70 \\
\hline Sondeo 3 & Capa 1 & & & & 0 & 27 & 1 & & & & & & 1 & 30 \\
\hline Sondeo 4 & Capa 1 & & & & 0 & 26 & & 4 & & & & & & 30 \\
\hline Sondeo 5 & Capa 1 & 24 & 18 & & 42 & 52 & 2 & 38 & & & 1 & & & 96 \\
\hline $\begin{array}{l}\text { Sondeo } 5 \\
\text { anexo a }\end{array}$ & Capa 1 & 7 & 6 & & 13 & 3 & & 14 & & & & & & 17 \\
\hline $\begin{array}{l}\text { Sondeo } 5 \\
\text { anexo b }\end{array}$ & Capa 1a & 3 & & & 3 & 2 & & 2 & & & & & & 4 \\
\hline $\begin{array}{l}\text { Sondeo } 5 \\
\text { anexo b }\end{array}$ & Capa 1b & 6 & 6 & & 12 & 27 & & 17 & & & & & 2 & 45 \\
\hline Sondeo 6 & Capa 1a & 30 & 8 & & 38 & 64 & 1 & 19 & & 1 & & 1 & 2 & 88 \\
\hline Sondeo 6 & Capa 1b & 21 & 8 & & 29 & 53 & 1 & 23 & & & 1 & & & 80 \\
\hline Sondeo 6 & $\begin{array}{l}\text { Capa 1a sobre } \\
\text { cenizas }\end{array}$ & 27 & 12 & & 39 & 107 & 4 & 64 & & & 3 & & & 185 \\
\hline Sondeo 7 & Capa 1 & 10 & 4 & & 14 & 29 & & 36 & & & 1 & & & 67 \\
\hline Sondeo 8 & $\begin{array}{l}\text { Capa 1a (con } \\
\text { bosta de oveja) }\end{array}$ & 32 & 7 & & 39 & 33 & 2 & 20 & & & & 1 & 1 & 61 \\
\hline Sondeo 8 & $\begin{array}{l}\text { Capa 1a (sin } \\
\text { bosta de oveja) }\end{array}$ & 6 & 3 & & 9 & 8 & & 15 & & & & & & 23 \\
\hline Sondeo 8 & $\begin{array}{l}\text { Capa de } \\
\text { cenizas }\end{array}$ & 11 & & & 11 & 8 & & 5 & & 1 & & 1 & 2 & 18 \\
\hline Sondeo 8 & $\begin{array}{l}\text { Capa } 1 b \text { (con } \\
\text { cenizas) }\end{array}$ & 4 & 5 & & 9 & 5 & & 7 & & & & 1 & & 14 \\
\hline Sondeo 8 & $\begin{array}{l}\text { Capa } 1 b \text { (sin } \\
\text { cenizas) }\end{array}$ & 2 & 1 & & 3 & 9 & 1 & 28 & & & & & & 39 \\
\hline Sondeo 9 & Capa 1a & 2 & 1 & & 3 & 4 & & 8 & & & & & 1 & 12 \\
\hline Sondeo 10 & $\begin{array}{l}\text { Capa 1a(sobre } \\
\text { cenizas) }\end{array}$ & & 1 & & 1 & 3 & & & & & & & & 3 \\
\hline Sondeo 10 & $\begin{array}{l}\text { Capa 1b } \\
\text { (ceniza) }\end{array}$ & 11 & & & 11 & 14 & & 3 & & & & & & 17 \\
\hline Sondeo 11 & Capa 1a & 17 & 26 & & 43 & 15 & 1 & 15 & & & 1 & & & 34 \\
\hline Sondeo 11 & Capa 1b & 25 & 20 & & 45 & 9 & & 16 & & & & & & 25 \\
\hline Sondeo 11 & Capa 1b & 9 & 10 & & 19 & 8 & & 10 & & & 3 & & & 24 \\
\hline \multirow[t]{2}{*}{ Anexo c } & Capa 1 & 1 & 2 & & 3 & 4 & & 4 & & & & & & 8 \\
\hline & Totales & 255 & 140 & & 395 & 567 & 17 & 379 & & & & & & 1018 \\
\hline
\end{tabular}

* C. ind.: cerámica indígena C. EU: cerámica europea. 


\section{BIBLIOGRAFÍA}

ARRIGONI, G. y C. PALEO. 1991. Investigaciones arqueológicas en la región central del golfo San Jorge (desde Punta Peligro, Pcia. del Chubut hasta el límite con la Pcia. de Santa Cruz). Shincal 3, (T.3): 206-210. Catamarca.

BARBERENA, R. G., L' HEUREUX y L.A. BORRERO. 2004. Expandiendo el alcance de las reconstrucciones de subsistencia. Isótopos estables y conjuntos arqueofaunísticos. En: Contra Viento y Marea. Arqueología de Patagonia, compilado por M.T. Civalero, P. Fernández y A.G. Guráieb, pp. 687-700, Instituto Nacional de Antropología y Pensamiento Latinoamericano y Sociedad Argentina de Antropología. Buenos Aires.

BORRERO, L.A. y S. CAVIGLIA. 1978. Estratigrafía de los concheros de Bahía Solano: campaña 1976-77. Comunicación presentada en el V Congreso Nacional de Arqueología Argentina, San Juan. (MS.)

BORRERO L.A., N. V FRANCO., R. BARBERENA, F. BORELLA, P. CAMPAN, F.CARBALLO MARINA, I. CRUZ, C. FAVIER DUBOIS, R. A.GUICHÓN, L. L'HEUREUX, M. V. MANZINI., L.MANZI y F.M. MARTIN. 2006. Arqueología en Cabo Vírgenes y Cañadón Gap. En Arqueología de la costa patagónica. Perspectivas para la conservación. Editado por: CRUZ, I y M.S. CARACOTCHE. Pp. 213-228 . Universidad Nacional de la Patagonia Austral. Río Gallegos.

CARACOTCHE, M.S., I. CRUZ, S. ESPINOSA, F. CARBALLO MARINA y J.B. BELARDI. 2006. Rescate arqueológico en el Parque Nacional Monte León (Santa Cruz, Argentina). Magallania 33 (2): 143-164. Punta Arenas.

CASTRO A., MORENO J., M. ZUBIMENDI, M. ANDOLFO, B. VIDELA, P. AMBRUSTOLO, L. MAZITELLI. 2007. Cronología de la Ocupación Humana en la Costa Norte de Santa Cruz: Actualización de datos radiocarbónicos. En: Arqueología del Fuego-Patagonia. Levantando piedras, desenterrando huesos... y develando arcanos. Editado por: F. Morello; M. Martinic; A. Prieto y G. Bahamonde. Pp. 527-534. Ediciones. CEQUA. Punta Arenas. Chile.

CAVIGLIA, S. y L.A. BORRERO. 1978. Bahía Solano: su interpretación paleoetnozoológica en un marco regional. Comunicación presentada en el V Congreso Nacional de Arqueología Argentina, San Juan. (MS.)

CAVIGLIA, S., L.A. BORRERO, M. CASIRAGHI, L.C. GARCÍA y V. HORWITZ. 1982 Nuevos sitios arqueológicos para la región de Bahía Solano (Chubut). Comunicación presentada en el VII Congreso Nacional de Arqueología Argentina, San Luis. (MS.).
CORDERO OTERO, R., C. M FAVIER DUBOIS y H. PANARELLO. 2000. Efecto reservorio en las dataciones por 14C sobre moluscos actuales de la costa patagónico-fueguina. Importancia arqueológica. En Desde el País de los Gigantes. Perspectivas arqueológicas en Patagonia. Pp.541-548, Universidad Nacional de la Patagonia Austral, Río Gallegos.

CRUZ, I. y M.S. CARACOTCHE (editoras). 2006. Arqueología de la costa patagónica, perspectivas para su conservación. Universidad Nacional de la Patagonia Austral, Río Gallegos.

EMBON, A. 1950. Fuentes históricas con noticias etnográficas y arqueológicas del indígena patagón. Tesis de Doctorado en Historia, Facultad de Humanidades y Ciencias de la Educación, Universidad Nacional de La Plata. (MS.).

EUGENIO, E.O. y V. ALDAZÁBAL. 2004. Los cazadores-recolectores del litoral marítimo del área de Bahía de San Blas, Provincia de Buenos Aires. En Contra Viento y Marea. Arqueología de Patagonia, compilado por M.T. Civalero, P. Férnández y A.G. Guráieb, pp. 687-700, Instituto Nacional de Antropología y Pensamiento Latinoamericano y Sociedad Argentina de Antropología. Buenos Aires.

GÓMEZ OTERO, J. 1995. "Bases para una arqueología de la Costa Patagónica Central (entre el Golfo San José y Cabo Blanco)". Arqueología 5 :61-103. Buenos Aires.

GÓMEZ OTERO J. 2007. Dieta, uso del espacio y evolución en poblaciones cazadoras-recolectoras de la costa centro - septentrional de Patagonia durante el Holoceno medio y Tardío. Tesis doctoral. Facultad de Filosofía y Letras. Universidad Nacional de Buenos Aires. MS.

GOMEZ OTERO, J. y F. SUAREZ. 1999. Lobos marinos y guanacos: Análisis arqueofaunisticos de un fogón hallado en la costa del Golfo San Matías, Península Valdés (Chubut). En: Libro de resúmenes del XIII Congreso Nacional de Arqueología Argentina. Pp. 396. Córdoba.

GOMEZ OTERO, J., H. MARANI e I. PEREZ. 2002. Aprovechamiento de guanacos en Península Valdés. Estudio arqueofaunístico del sitio La Armonía (Muestreo 2). En: Intersecciones en Antropología 3: 17-29, Facultad de Ciencias Sociales, Universidad Nacional del Centro de la Provincia de Buenos Aires, Olavarría.

MANSUR, E. 2007. Confección y uso de artefactos discordes en contextos de cazadores recolectores de Patagonia Meridional: pesas para redes en el sitio HST01AM (Prov. Santa Cruz), Argentina. En: Arqueología del Fuego-Patagonia. Levantando piedras, desenterrando huesos... y develando arcanos. Editado por: F. Morello; M. Martinic; A. Prieto y G. Bahamonde. Pp. 701-708 Ediciones. CEQUA. Punta Arenas. Chile. 
MARTINIC, M. 1995. Los Aónikenk. Historia y cultura. Ediciones de la Universidad de Magallanes, Punta Arenas.

MARTINIC, M. y D. QUIROZ. 1989-90. El uso ecuestre entre los Aonikenk. Anales del Instituto de la Patagonia (Serie Ciencias Sociales) 19:29-42. Punta Arenas.

MASSONE, M. 1979. Panorama etnohistórico y arqueológico de la ocupación tehuelche y proto-tehuelche en la costa del Estrecho de Magallanes. Anales del Instituto de la Patagonia (Serie Ciencias Sociales) 10: 63-107. Punta Arenas.

MORENO, J.E. 2003. El uso indígena de la costa Patagónica Central en el Período Tardío. Tesis Doctoral. Facultad de Ciencias Naturales y Museo. Universidad Nacional de La Plata. (MS.)

MORENO, E. y CASTRO A. 1995-96 La Costa Norte de Santa Cruz como excepción al modelo de cazadores de guanacos. Anales de Arqueología y Etnografía N ${ }^{\circ}$ 50-51:13-22. Instituto de Arqueología y Etnografía, Facultad de Filosofía y Letras. Universidad Nacional de Cuyo. Mendoza.

MORENO, E., A. IZETA. 1999 Estacionalidad y Subsistencia indígenas en Patagonia Central según los viajeros de los Siglos XVI-XVII. Soplando en el viento. Universidad Nacional del Comahue, Neuquén, Argentina Pp. 477-490.

ORQUERA, L. A. 1986. Avances en arqueología de Pampa y Patagonia. (Buenos Aires, fotoduplicación). Trad. del trabajo en: Advances in Wolrd Arqueology 6. Academic Press.

PALERMO, M. A. 1988. La innovación agropecuaria entre los indígenas pampeanos-patagónicos. Génesis y procesos. Anuario del IEHS 3: 43-91. Universidad del Centro de la Provincia de Buenos Aires. Tandil. 